\title{
FUSION OF MULTIMEDIA SMART GAMIFICATION AND GAMES WITH APPLICATION IN MENTAL HEALTH
}

\section{Andreja Samčović ${ }^{\star}$ Svetlana Čičević, Slobodan Mitrović}

Faculty of Transport and Traffic Engineering, University of Belgrad, Belgrade, Serbia

\begin{abstract}
:
Serious games and smart gamification potentially represent an efficient combination of smart systems and technologies on the one hand, while on the other hand they represent a combination of mechanics used for computer games in serious fields of application and gamification. The capabilities and advantages of both fields are combined and as a result they get help from users who need it.This paper describes their application in mental health, as an important issue for all of us.
\end{abstract}

Keywords:

gamification, serious games, smart objects, smart systems, mental health.

\section{INTRODUCTION}

During the last decade, we are witnesses to anenormous growth in the field of computertraining systems, which allow anintegration of multimedia, serious gaming,and smart technologies in different areas such as education or health [1]. Such techniques utilize recent advances in virtual reality (VR) and augmented reality (AR) to form three-dimensional (3D) virtual models with avatars, which increase the usability of these platforms. Serious gaming includes methods and conception of game technology (multimedia, VR, AR, computer graphics, etc.) with various areas, such asinformation and communication technologies (ICT), psychology, design, etc. We must point out "serious areas of applications" such as learning,militarysituations, or health applications, among others [2].

The rapid growth of smart connected systems and smart technologies enables users to implement new approaches to solve different requirements, and reduce time required to provide better Quality of Service (QoS) [2]. On the other hand, the development of serious games and game-related technologies stimulated research in serious areas such as e-health, education, military and other fields. One definition of gamification includes the usage of various objects to design games in contexts unrelated to games [3, 4].
Correspondence:

Andreja Samčović

e-mail:

andrej@sf.bg.ac.rs 
While serious games are not used for entertainment purposes, gaming applications use game elements but never reach the level of being called a game. Along with serious games, gamification utilizes games for purposes other than entertainment. Having that in mind, gamification represents the active implementation of concepts for serious games, game analytics, computer game technology, and so on, to enhance customer skills and experience [2].

Recent advances on smart systems, smart technologies, gamification, gaming applications and serious games show that these areas will be very important in the following years and are expected to be broadly accepted by users. The aim of this paper is to understand the upcoming smart gaming technologies and serious games, to make classificationof main components and technologies on which they are based, and to formulate the requirements for design and development of smart applications.

We can say that researches on this topic should:

- Describe the present position smart systems and smart technologies, as well as gaming applications and serious games;

- Analyze and classify smart objects and their properties, as well as the their levels of intelligence;

- Analyze and classify smart gaming and serious games, their technologies, features and characteristics;

- Show the differences between serious games and smart gaming applications;

- Provide opportunities for merging gaming and smart systems/technologies into smart gaming, as well as serious games with smart systems/technologies into smart serious games [2].

This paper is organized as follows: the first second section after introduction describes with smart objects, technologies and systems, as well as their classification. Gamification and Smart Serious Games (SSG) are presented in the next part, followed by describing their application in very important field mental health. Finally, the last section concludes the paper.

\section{SMART SYSTEMS, FACILITIES AND TECHNOLOGIES}

Smart systems/technologies with different types of intelligence are already used today.An example of a smart object is smart material. It can detect sensitivity changes depending of environmental parameters, individual signals, or even restore degraded sensitivity [2]. An example of a smart textile material is a smart T-shirt. One application of a smart T-shirt is related to soldiers. If a soldier is hit by a bullet, the smart T-shirt detects a number of signals from the soldier's body based on how urgent the response is (whether the wound is dangerous and must be urgently intervened or not).

An example of smart technology is smart home technology. Smart home technologies enable users to use energy efficiently in their homes, and to use home resources to make their life easier, as well. They allow temperature control by monitoring its changes e.g. devices such as if air conditioning are switched on/off on the command of smart home technology. The other example of smart home technology is room lighting that can also be controlled. This control regulates energy consumption and reduces costs, which is important for green technologies. Also, an integral part of the smart home is the anti-theft system. One example of a smart activity is the smart shopping list application supported by mobile phones.

\section{Smart entity classification}

Smart entities can be divided into:

- Smart systems (smart phones, smart cities, smart classrooms...);

- Smart objects (smart sensors, smart materials, smart medical devices...);

- Smart activities (smart learning, smart gaming, smart shopping...)

- Smart technologies (smart sensors, smart RFID (Radio Frequency Identification), smart grid technologies...)

Smart entity features include:

- Collecting raw data from the real world (environment) using sensors, which facilitate environmental monitoring. The obtained data is converted and thus saved for further processing;

- Transmission of the collected data by sensors to the control unit by means of transmitters;

- Big data to manage the system appropriately as determined by the processing of the previously obtained data by the sensor;

- Transmission of instructions from the processing unitsto other devices in the network, via internet;

- Activation of real and/or virtual actuators in response to the obtained data. 
Taking it into account, we can classify the main features of a smart system:

- Objective - Use of sensors, infrastructure components, processes, technologies, to obtain quality information, can help users to make effective crucial decisions at the right time;

- Components - Smart objects, smart technologies, smart services, and corresponding subsystems;

- Connections-Radio/electrical, physical, thermal, infrared and internet based connections;

- Input size - Large amount of raw data and significant environmental information such as sensor data;

- Output size - Recommendations based on information, immediate execution of certain actions using software systems and information technologies, web services, actuators, and so on;

- Interfaces - Internet Protocols (IP), RFID Protocols;

- Limitations - weight, type, size, processing time 2of raw data, and so on;

- Environment.

\section{Smart System Intelligence Levels}

In order to highlight the differencesamong smart systems by which one is smarter, we can use classification by intelligence levels and smart technology skills:

- Adaptation - The ability to change physical characteristics or behavior to adapt to the environment;

- Sensitivity - The ability to detect, understand, recognizeand/or perceive a phenomenon, event, or object;

- Inference ability - The ability to make logical inferences based on raw data, or information based on specific rules;

- Learning ability - The ability to acquire new skills or to change existing to improve performance;

- Expectation - The ability to predict what will happen or what to do next;

- Self-organization capability - This is the ability of a system to modify its components, or to refresh in certain circumstances without an external agent or entity.

Definition of smart games: software that combines a "gaming" dimension (concept, design and model of games), game structure, gametechnique and graphical user interface) [5]. Classification of smart gaming is important for better understanding of the purpose, domain, type of game, and as a result, effective fusion of smart technology with smart games.
The purpose of smart games can be different, such as broadcasting messages (educational, so-called edugames, persuasive advergames, informative newsgames, military games and art games), physical and mental training and data exchange $[6,7]$.

Scope of smart games may consist of market/domain codes (such as state and government, military, public health, education, corporation, art, scientific research and entertainment) and general public, experts and students [2]. Smart games can be game-based (with a defined aim to play), and play-based (aimless to play).

\section{GAMIFICATION}

Gaming can be defined as the process of incorporating gaming dimension - game design, structures, gamelike graphical user interface into an existing non-fun dimension (such as a software system, corporation or learning community, customer community, web-site, etc.) to motivate professional development and personal productivity growth and efficiency, quality of learning and training, active participation and engagement, and/ or long-term loyalty [2].

It is generally accepted that gamification can increase user participation and encourages distinctive unique experiences, as it increases access to other services and opportunities, enabling the participants to gain information for active interaction with other users. Because of the benefits of providing information and knowledge using game design and development, gamification has a lot of advantages in practice.Since gamification is a relatively new concept, it is largely uncertain how an effective design can be accepted, and there is limited knowledge on how to a variety of systemswill take advantage of gamification [8].

Gaming mechanics included into a gaming application or software can have animportantinfluence on the user or player type, who may or may not use those techniques in an efficient way. Significantvendors including Microsoft, Google, Adobe, Oracle, Cisco, Siemens, Ford...have already implemented gamified techniques and applications in supporting core functions, processes and/or activities [2].

Moreover, advantages of using smart games in business processes increase positive psychology of workers, strengthens and include the following $[2,9]$.

- Positive emotions (joy, pleasure, fun, safety, etc.);

- Engagement (continuous involvement in activities); 
- Relationships (mutual supportive interactions);

- Meaning (creating a meaningful narrative);

- Achievement (achievement of goals, monitoring of basic value).

In addition, smart games on the web-sites promote: good retention and effective memory factors, high information adaptability, more interactivity, attractive and unobtrusive promotion, and higher 'virality'. Table 1 presents one possible classification of the smart serious games (SSG) [2].

Table 1. Proposed classification of the smart serious games

\begin{tabular}{|c|c|c|}
\hline $\begin{array}{l}\text { SSG } \\
\text { feature }\end{array}$ & SSG characteristics & SSG details (types, classes) \\
\hline \multirow[t]{15}{*}{ Purpose } & Decision making & $\begin{array}{l}\text { Instruments for faster and } \\
\text { better decision making }\end{array}$ \\
\hline & \multirow[t]{2}{*}{ Simulation } & $\begin{array}{l}\text { Face-to-face or simulation } \\
\text { of real situations }\end{array}$ \\
\hline & & $\begin{array}{l}\text { Subjective } \\
\text { (military games, art games) }\end{array}$ \\
\hline & \multirow{2}{*}{$\begin{array}{l}\text { Knowledge } \\
\text { sharing }\end{array}$} & Edugames \\
\hline & & $\begin{array}{l}\text { Informative games } \\
\text { (news games) }\end{array}$ \\
\hline & Influence to do & Persuasive games \\
\hline & \multirow{3}{*}{$\begin{array}{l}\text { Data } \\
\text { capture/exchange/ } \\
\text { exploration }\end{array}$} & $\begin{array}{l}\text { Games based on data } \\
\text { capture and exchange }\end{array}$ \\
\hline & & $\begin{array}{l}\text { Focus on } \\
\text { research\&innovation }\end{array}$ \\
\hline & & Adventure games \\
\hline & Motivation & $\begin{array}{l}\text { Badgesand rewards points, } \\
\text { included in work }\end{array}$ \\
\hline & \multirow[t]{5}{*}{ Training } & Focus on analytical skills \\
\hline & & Focus on technical skills \\
\hline & & $\begin{array}{l}\text { Focus on management } \\
\text { skills }\end{array}$ \\
\hline & & $\begin{array}{l}\text { Focus on communication } \\
\text { skills }\end{array}$ \\
\hline & & $\begin{array}{l}\text { Focus on team working } \\
\text { skills }\end{array}$ \\
\hline \multirow[t]{12}{*}{ Scope } & \multirow[t]{12}{*}{ Domain } & Corporation \\
\hline & & Education \\
\hline & & State and government \\
\hline & & Health care \\
\hline & & Marketing \\
\hline & & Military \\
\hline & & Culture and arts \\
\hline & & Ecology and environment \\
\hline & & Politics \\
\hline & & Advertising \\
\hline & & Humanitarian \\
\hline & & Research \\
\hline
\end{tabular}

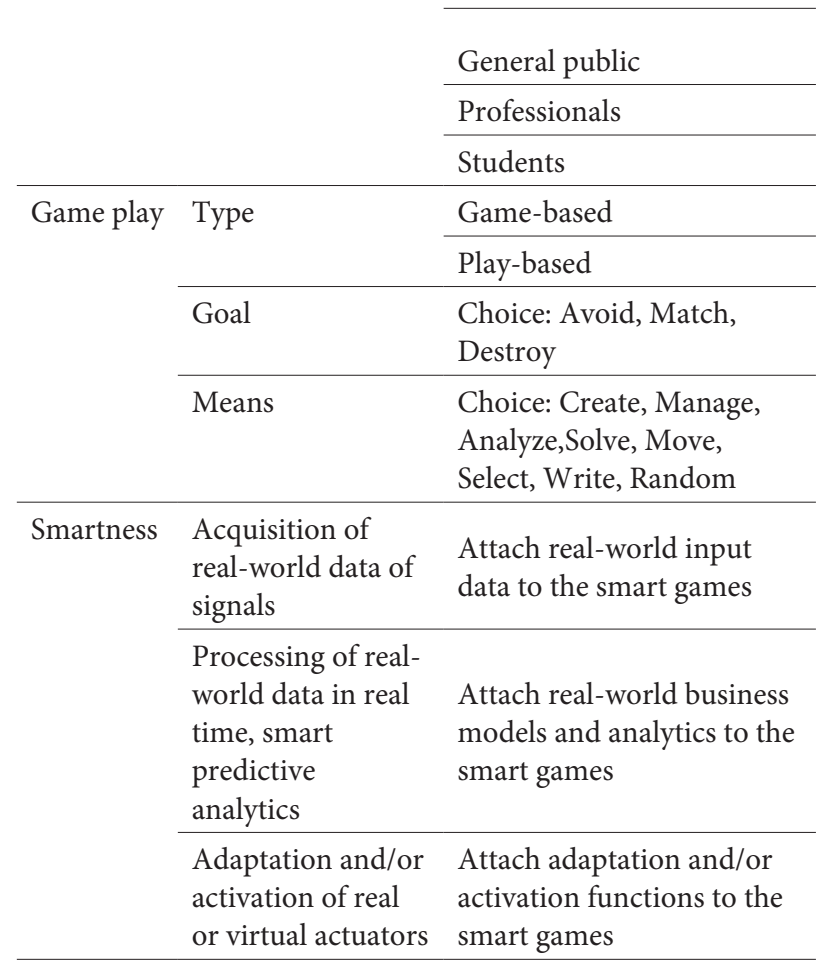

\section{SMART GAMIFICATION}

Smart gamification (SGM) is aimed at connecting smart systems, smart technologies, smart sensors, etc. Gamification features include: game mechanics, game design/methodology, game analytics, and graphical user interfaces.

Set of intellectual features that can be implemented consists of:

- Collecting (reading) of raw data or signals, or possibly locally processing the raw information [2];

- Transfer of raw and/or pre-processed sensor information;

- Data processing and detailed analysis of obtained information (smart analytics);

- Transfer of instructions;

- Activation of real and/or virtual actuators (software systems and tools).

The research, development, and wide acceptance of SGM and SG in societywould show the way to the convergence of physical and virtual society. The results of the research community allowedto showthe expected tendency of development in the field of SGM and SG.

Effective coupling of smart systems and smart technology with gaming techniques and serious games will be established on the addition of smart games characteristics, gaming and gamified applications. 
Smart characteristics of SG and SGM - customization, feeling, learning, anticipation, self-organization will connect users with the environmental issues, different activities, and better solutions [2].

SGM and SG will be developed into "smart" instruments to help users in solving "serious" problems. This is because SGM and SG will actively use sophisticated virtualization techniques, computer technologies, advanced video games, as well as smart systems and technologies [10]. SGM and SG can help users to focus on real-world scenarios and situations, applying various methods to potential solutions, as well as changing/enhancing their behavior and skills [2].

SGM and SG analytics can help to interpret and process big data produced by computer information systems, to improve production, security monitoring and other systems. In addition, they will help them to make decisions based on data that minimize potential risks. It will also allow the presentation of relevant findings and outcomes in a simplified way. Users who are not experts in this field can optimize gaming techniques to increase their productivity, efficiency, motivation, behavior, etc.

SGM and SG will influence the development of smart tools that significantly increase customer engagement. Key components of smarter user engagement are: (a) research (curiosity, search, gathering, analyzing ...), (b) competition (challenges, winning, coaching, learning), (c) collaboration (teamwork, assistance), (d) expression (creativity, design), and (e) being smart (inference, foresight, self-organization..)

The active implementation and use of SGMs and SGs will enable significant benefits. Some of the benefits are: (a) improving the performance, productivity, (b) comfortable quality training and acquiring new skills and knowledge, (c) moving staff through smarter health initiatives, (d) insight into customers and business partners, (e) customer engagement as part of a long-term relationship to develop a strategy, and (f) innovation through the mutual insight of the Internet of Things (IoT) $[2,11]$.

SGM and SG will reinforce positive organizational psychology. They will strengthen and expand PERMA concepts including: (a) Positive emotions, (b) Engagement, (c) Relationships (pleasant interactions with other users), (d) Meaning (producingmeaningful narration), and (e) Achievement of goals.SGM and SG will be firmly incorporated into new kinds of mobile, social and virtual systems and technical platforms.

\section{SERIOUS GAMES AND MENTAL HEALTH}

In this section, the prospective of gamification and serious games for mental healthadvancement will be presented. Serious games show a positive impact of internet technologies in mental health improving by employing three different processes. First processis offeringthe online applications to users who otherwise would not have a chance to use them [12]. Second process is improvement of users' engagement during game based and "serious" motivation. Third, by using various mechanisms for changing behavior and various kinds of learning including therapeutic processes [12].

Having that in mind we can distinguish five different types of serious games in mental health improvement. There are, as following: virtual and augmented reality, exergames, Cognitive behavior therapy, Cognitive training games, and Serious Motion-Based Exercise Games for Older Adults[13]. It was shown that traditional approaches can be replaced with computer approach, and to avail characteristics of serious games for remedial purposes [12]. We can conclude that serious games show significant potential to improve mental health with advanced internet technologies [12].

Dimensions of serious games-goals, interaction, and involved technologies showtremendous variations. They encompasses many different types from fast-paced mini-games(e.g., simple as stacking dots in a row), through Applied Games for Mental Health to augmented reality (AR), and complex shared worlds. Quality serious games have been shown to improve concentration (3), improve information retention (4), facilitate deep learning (5), and lead to behavior change (6) [12].

Although the application of serious games in areas such as psychological and behavioral changes or symptom relief is still in its infancy stage there are indices that they can be useful. While barriers still exist, there has been a significant increase in adoption of smartphone applications for mental health.

It is first necessary to point out that applied gaming offers potential in mental health, as given the popularity of serious games suggests. Applied approaches might be able to help improve many of the mental health interventions for people who might not otherwise be able to access help. Games play an important role for personsthat suffer from mental health problems and still are not treated (gap in treatment of mental health). 
Furthermore, applied gaming has attractive possibility. People experience serious games as funny, want to "win" the game, or see how the story unfolds. Such dynamics can influence to the reduction of high exhaustion rates in naturally conducted internet interventions.

Next, applied gaming has a capability for efficiency, because it has the opportunity for both traditional and non-traditional behavioral and learning that can be applied. For example, applied gaming can provide real experiences in which the "flow" state can be achieved, support sensory environments that could offer assistance for learning, provides behavioral models and social learning, enable users to try new abilities in a safe but reactive environment, and rehearsing to teach a new behavior.

Further escape motivation has also been reported (where a user needs to play to avoid real-world problems). A user may have high motivations for game playing, and the main motivations to play game may vary depending on demographic characteristics, contexts, and types of games [2].

We will explain now five main types of applied games, which can be found in the open literature [2]. It should be pointed out that they can provide opportunities by reaching large amount of people.

Virtual Reality (VR) and Augmented Reality (AR) are an interactive and immersive experiences in the virtual or augmented world, with visual, audio, and with sensory modalities to increase engagement of users and possibly therapeutic influence [2].

Exergames are sport games or games based on movements. There is a significantlarger effect of playfulness in exergamesfor depressive symptoms GAMES FOR HEALTH, with it being more attractive among other playful games, over those that involving less game elements [14, 15].

Cognitive behavior therapy $(\mathrm{cBt})$ is an efficacious treatmentwhich uses serious games and gamification that often use an adorable environment, and designed at a rate of one level weeklyon average on a computer. Each of these therapeutic programs was created for children or young people [14]. One example of those games is SPARX SuperBetter narrative, computer based serious game.Another example is SuperBetter,which offers anotherapproach to gamification, with rewards \&scoring joy serious games have impact on mood. Users playing a violent serious game reported reduced depressive symptoms immediately after the interference of the serious game Bejeweled II [12]. It is suggested to be employed in this visually spatial cognitive-behavioral activity when traumatic memories are activated to help preventing flashbacks [2].
Cognitive training games - It would be helpful to examine popular types of games for target audience and compare their important characteristics with those features used in mental health games. For example, highaccess games presently involve mobile gaming on smart phones, numerous multiplayer serious games in which many players interact, games that allow user-generated media content, and games related to main social media platforms [2].

Popular among marketing and learning environments, gamification also pays attention to health care. Evidence suggests that gamification raises enjoyment, engagement and compliance of health related activities [16], while positively impactingboth the cost of service provisionand health outcomes. Its implementation has been enhanced by the development of smartplatforms based on digital health, created around the ecosystem of wearable devices for fitness purpose (such as Fibit) or other sensing devices such as smartphones [17].

Gamification has great potential to add additional positive experiences to their primary health goals. Mentioned digital services can be called as Health Behaviour Change Support Systems (HBCSS), while their goal is to change the belief and behaviour of individuals towards well-being and a healthier lifestyle. Therefore, the main assumption of HBCSS gamification is that behaviour and attitudes of humans can have a positive influence through communication interventions [17]. These attitudes and behaviours need to havetime to be maintained in order to offer concrete and positive health and well-being results. In this sense, the temporal dimension inside gamification is of utmost importance [17].

They aim, through artifacts, to induce behavioral change. The main areas of application of gamified HBCSS are the health promotion, physical activity, dietary guidance, as well as supportingthe health practitionerswithin their education and daily activities [17].

Serious Motion-Based Exercise Games for Older Adults - Ambient Assisted Living (AAL) is a field to involve communication technology, sensors, actuators, into the real environment to improve safety and security, the everyday life, and independence in older adults in order to facilitate aging in homes. Examples of AAL technologies include sensors invisibly integrated into the system to detect falls, supportive prescribed medication, communication channels to members of family or medical nurses, and home service robots to support elderly care. However, theiraim is to ensure that aging technology is aligned with the needs of older adults and, most importantly, in the motivation for use to adopt it [13]. 
The natural development of computer applications is moving towards mobile applications that can be used on a smartphone.Smartphone mental health applications can include many of the benefits, such as cost-effectiveness, plus they are always online, almost always with a person, and can collect data through their integrated sensors.

Last, but not least, serious games designed for their psychotherapeutic properties have been used for, among others, to treat depression, impulse-control disorders, schizophrenia, and anxiety disorders [18].

\section{CONCLUSION}

We presented in this paperthe possibilities of gamification and multimedia serious games as relatively new fields with en emphasize in mental health which can help numerous users. This application could be potentially very applicable in the recent pandemic, such as COVID-19. This area is accessible for future improvement, taking into account various approaches. Future survey should compare options that are game based and non-based for different groups of users.

\section{ACKNOWLEDGMENT}

This paper is partially supported by the Ministry of Education, Science and Technological Development of the Republic of Serbia.

\section{REFERENCES}

[1] M.Favorskaya(et al.:): "Advances in Smart, Multimedia and Computer Gaming Technologies", in "Fusion of smart, multimedia and computer gaming technologies", Springer, pp 1-6, 2015.

[2] Sharma, M. Favorskaya, L. Jain, R. Howlett (eds.): "Fusion of smart, multimedia and computer gaming technologies", Springer, 2015.

[3] S. Deterding, R. Khaled, L. Nacke, D. Dixon: "Gamification: toward a definition", in ACM Workshop CHI'11, Vancouver, Canada, 2011.

[4] [mafiadoc.com]

[5] Uskov, B. Sekar: "Serious games, gamification and game engines to support framework activities in engineering: case studies, analysis, classification and outcomes", in Conference on electronics/information technology EIT-2014, Milawaukee, WI, USA, pp. 618-623, 2014.
[6] A. Jaume-i-Capo, A. Samčović: „Interactive multimedia system using serious game for users with motor disabilities", Telfor Journal, Vol. 7, No. 2, pp 97-102, 2015.

[7] A. Samčović: „Serious games in military applications", Vojnotehnički glasnik, Volumen 66, broj 3, str. 597-613, Beograd, jul-septembar 2018.

[8] D. Djaouti, J. Alvarez, JP Jessel: "Clasifying serious games: the G/P/S model”, in P. Felicia (ed.), Handbook of research on improving learning and motivation through educatiol games: multidisciplinary approach, IGI Global, Hershey, 2011.

[9] B.C. Lee: "The effect of gamification on psychological and behavioral outcomes: implications for cruise tourism destinations", Sustainability, Vol. 11(11), paper 3002, May 2019.

[10] P. Boinaliris, P. Fingar: "Serious games for business using gamification to fully engage customers, employees and partners", Meghan-Kiffer Pres, Florida, 2014.

[11] D. Drajić: "Uvod u IoT (Internet of Things), Akademska misao, Univerzitet u Beogradu Elektrotehnički fakultet, Beograd, 2017.

[12] A. Uskov: "Gamification in computer science", IIMSS, 2013.

[13] [games.jmir.org]

[14] T.M. Fleming, et.al.: "Serious games and gamification for mental health: current status and promising directions", Frontiers in Psychiatry, Vol. 7(215), January 2017.

[15] J. Li, Y.L. Theng, S. Foo: "Exergames for older adults with subthreshold depression: Does higher playfulness lead to better improvement in depression?", Games for Health Journal, Vol. 5, No. 3, pp 175-182, June 2016.

[16] E. Duarte, P. Pereira, F. Rebelo: "A review of gamification for health-related contexts", International Conference of Design, User Experience, and Usabiility, June 2014.

[17] S. Stepanović, T. Mettler: "Gamification applied for health promotion: does it really foster long-term engagement? A scoping review", $26^{\text {th }}$ European Conference on Information Systems ECIS 2018, pp. 1-16, Portsmouth, UK, 2018.

[18] P. Brauner, M. Ziefle: "Serious motion-based exercise games for older adults: evaluation of usability, performance, and pain mitigation",JMIR Serious Games, Volume 8, No. 2: e14182, 2020. 